\title{
Igualdad y mérito ¿un conflicto de valores? ${ }^{1}$ Equality and Desert, a Conflict of Values?
}

\author{
Olof Page
}

Instituto de Filosofía, Pontificia Universidad Católica de Chile

\begin{abstract}
Resumen. Desde hace al menos tres décadas el concepto de igualdad —en particular, el concepto de igualdad distributiva- ha estado en el centro de la discusión contemporánea sobre justicia distributiva. La idea que las desigualdades inmerecidas deberían ser eliminadas y que las desigualdades merecidas deberían ser preservadas es una de las maneras más relevantes a través de la cual el ideal de la igualdad ha sido recientemente defendido. El objetivo principal de este trabajo es argumentar contra el valor intrínseco de la igualdad entendida de esta manera apuntando a una importante confusión: aquélla entre el valor de la igualdad y el valor de que las personas tengan lo que merecen.
\end{abstract}

Palabras clave: mérito, igualdad, oportunidades, responsabilidad, suerte.

\section{Consideraciones previas}

En materia de justicia distributiva existe una importante corriente de pensamiento que considera que el foco de nuestra atención moral debería estar puesto en aquellas desigualdades que no son responsabilidad de las personas. Desde este punto de vista sería moralmente malo que algunos estén peor que otros por razones que no tienen que ver con su responsabilidad sino que con la suerte. Introducir la responsabilidad como factor a considerar permitiría diferenciar entre tipos de desigualdad moralmente relevantes. Las desigualdades que no son
Abstract. Since at least three decades, the concept of equality -in particular, distributive equality - has been at the centre of the contemporary discussion on distributive justice. The idea that underserved inequalities should be eliminated and that deserved inequalities should be preserved is one of the most relevant ways in which the ideal of equality has been recently defended. The main scope of this article is to argue against the intrinsic value of equality thus understood by pointing to an important confusion: the one between the value of equality and the value of people having what they deserve.

Key words: desert, equality, opportunities, responsibility, luck.

responsabilidad de las personas deberían ser corregidas y las que sí lo son no deberían serlo. Conocidos exponentes de esta concepción son, por ejemplo, G. A. Cohen, Ronald Dworkin y Thomas Nagel. G. A. Cohen piensa que «parte importante del propósito fundamental igualitario es extinguir la influencia de la suerte (brute luck) en la distribución. La suerte es un enemigo de la igualdad justa, y, ya que los efectos de la elección genuina contrastan con la suerte, la elección genuina excusa desigualdades que de otro modo serían inaceptables» (1989, 931). Ronald Dworkin piensa que los individuos deberían ser liberados de res- 
ponsabilidad «por aquellos elementos desafortunados de su situación debidos a la mala suerte (brute bad luck), pero no por aquellos que deberían ser vistos como fluyendo de sus propias decisiones. $\mathrm{Si}$ alguien nace ciego o sin los talentos que otros tienen, ésta es su mala suerte, $\mathrm{y}$, hasta donde esto pueda manejarse, una sociedad justa debería compensarlo por esa mala suerte. Pero si esta persona tiene menos recursos que otros porque gastó más antes en lujos, o porque decidió no trabajar, o trabajar en labores de más baja remuneración que las que escogieron los demás, entonces, su situación es el resultado de la elección, no de la suerte, y él no tiene derecho a ninguna compensación que pudiese mejorar su actual situación» (2000, 287). Thomas Nagel piensa que «lo que parece mal no es que las personas deban ser generalmente desiguales en ventajas o desventajas, sino que deban ser desiguales en ventajas o desventajas por las cuales no son responsables. [...] Dos personas que nacen en una situación que les da iguales chances de vida pueden terminar viviendo vidas de muy distinta calidad como resultado de sus propias libres decisiones, y ello no debería ser objetable para un igualitario» (1991, 71).

Esta manera de entender la relación entre suerte, responsabilidad y justicia se conoce como «igualitarismo de la suerte» [luck egalitarianism] y, según algunos, es «una de las teorías de justicia distributiva más significativas emergidas desde la publicación de Teoría de la justicia» de John Rawls (Scheffler 2003, 5). ${ }^{2}$ Aunque coincido con ese juicio creo, sin embargo, que el igualitarismo de la suerte encierra importantes problemas. No es mi propósito, en todo caso, analizar aquí cuáles son todos esos problemas. ${ }^{3}$ Mi propósito es, más bien, discutir algunas de las razones que recientemente se han ofrecido para defender el valor intrínseco de la igualdad entendida de esta manera. En este ensayo argumentaré contra el valor intrínseco de la igualdad. Esta argumentación tendrá como marco de referencia el supuesto conflicto entre el valor de la igualdad y el valor del mérito. Como se verá, dada la concepción de la igualdad distributiva que nos interesa aquí, el conflicto entre igualdad y mérito es el escenario adecuado para esclarecer el valor de la igualdad.

Algunas aclaraciones previas. Primero, cuando hablo de igualdad (o de desigualdad), me refiero a igualdad (o desigualdad) de algún bien estimado relevante. No es mi objetivo aquí justificar la elección de algún bien o (conjunto de bienes) específico. Asumiré, por tanto, que, cuando hablamos de igualdad ya contamos con una respuesta a la pregunta ¿igualdad de qué? ${ }^{4}$ Esta aclaración sirve, entre otras cosas, para entender que no estamos hablando de cualquier tipo de igualdad sino sólo de igualdad relativa al bien o los bienes considerados relevantes. Por esta razón, cada vez que aquí se diga que alguien está peor o mejor que otro, debe entenderse que está mejor o peor en términos del bien relevante.

Segundo, cuando hablo de igualdad me refiero a la igualdad así como es entendida por los igualitaristas de la suerte. Esta manera de entender la igualdad se distingue de la igualdad estricta, es decir, de ese punto de vista que considera que toda desigualdad del bien relevante es moralmente objetable, independientemente de si hay o no responsabilidad individual de por medio. Me referiré a la igualdad que me interesa aquí como «igualdad no-estricta» o, cuando el contexto es claro, simplemente como «igualdad».

Tercero, cuando hablo aquí de mérito me estoy refiriendo al criterio moral según el cual se considera que un deter- 
minado agente, en virtud de una determinada razón - llamada «base de mérito»-, merece más o menos del bien relevante. Hay una cantidad importante de problemas relacionados tanto con la base de mérito como con el bien merecido que aquí no trataré. ${ }^{5}$ Supondré que existen razones que justifican adecuadamente el uso del mérito así entendido. Esto significa que no consideraré, por ejemplo, si estamos o no suficientemente determinados por contingencias naturales y sociales como para que los juicios de mérito carezcan de una justificación moral adecuada; o si puede existir algún tipo de compatibilidad entre determinismo y mérito que de algún modo salve la posibilidad de realizar esos juicios.

Cuarto, no voy a pronunciarme sobre al valor moral del igualitarismo de la suerte, es decir, si es o no moralmente deseable que se diga, por ejemplo, que, si una persona es responsable por estar peor que otra, entonces merece estar como está. Lo importante aquí es entender que, al hablar de desigualdades merecidas, me estoy refiriendo a desigualdades que pueden atribuirse a la responsabilidad de los involucrados, sin que esa atribución implique una evaluación moral de la desigualdad en cuestión. Es decir, cuando me refiero a las desigualdades por las cuales las personas son responsables como desigualdades merecidas no estoy diciendo con ello que la desigualdad merecida deba entenderse como un castigo por un comportamiento moralmente reprochable o como un premio por un comportamiento moralmente valioso.

Por último, cuando hablo aquí de «suerte» hago referencia a la ocurrencia impredecible de eventos o circunstancias que están fuera del control de las personas y que no son atribuibles a la acción de otros sujetos, es decir, circunstancias o eventos inevitables.

\section{El problema}

Parte del problema que motiva este trabajo es el siguiente. Parece razonable describir las desigualdades que no son responsabilidad de las personas como desigualdades inmerecidas y aquéllas que sí lo son como desigualdades merecidas. Puestas así las cosas, cabe preguntarse qué es lo que realmente le importa a esta concepción de la igualdad distributiva, si la desigualdad o el que las personas no tengan lo que se merecen. $\mathrm{Si}$ fuera esto último, entonces la igualdad no importaría en sí misma, es decir, no tendría un valor intrínseco, como algunos piensan. Esto es lo que justifica que se hable aquí de un posible conflicto entre igualdad y mérito. Para que este conflicto exista deberían darse, entonces, las siguientes condiciones: (i) la persona A está peor que la persona $\mathrm{B}$, (ii) A y B merecen estar como están. Por un lado, la preocupación por la desigualdad demanda ayudar al que está peor y, por otro, la preocupación por dar a cada uno lo que merece demanda - en este caso en particular - dejar las cosas como están. ¿Qué demanda debe prevalecer?

Parte de lo que sostendré en relación a este problema descansa en la tarea realizada por Shelly Kagan en un importante ensayo (Kagan 1999). La conclusión principal de dicho ensayo es que, en todos aquellos casos en los que la igualdad entra en conflicto con el mérito, las demandas normativas del mérito prevalecen. Esto muestra que la igualdad no tiene un valor intrínseco. Mi propósito es distinto del de Kagan en cuanto pretendo mostrar, primero, que, cuando entendemos el igualitarismo de manera no-estricta el mérito no sólo tiene mayor peso normativo que la igualdad - como pretende Kagan-, sino que nunca puede siquiera oponerse a ella y, segundo, que, si esto es correcto, no hay razón para 
limitar nuestra atención sólo a las desigualdades inmerecidas. Si nos preocupan las desigualdades inmerecidas debería también preocuparnos todo estado de cosas inmerecido, sea éste uno igual o desigual.

En un nivel más específico, mi intención es alcanzar este propósito revisando y criticando algunos argumentos contra Kagan que buscan, de diferentes maneras, defender el valor intrínseco de la igualdad. Estos argumentos contemplan, por un lado, la defensa del valor intrínseco de la igualdad entre individuos igualmente merecedores (Temkin 2000, 2003; Olsaretti 2002) y, por otro, la defensa del valor intrínseco de la igualdad de base (Olsaretti 2002). En el primer caso hablamos del valor de la igualdad entre individuos que están en condiciones de merecer (porque son sujetos responsables) y, en el segundo, hablamos del valor de la igualdad entre individuos que no están en condiciones de merecer (porque son, por ejemplo, recién nacidos). Pretendo mostrar que esos argumentos a favor del valor intrínseco de la igualdad no son buenos argumentos. Esto me permitirá esbozar, además, una conclusión que considero importante pero que, para ser desarrollada adecuadamente, requeriría de otro trabajo. Esta conclusión afirma que, quienes otorgan a la igualdad un valor intrínseco, confunden el valor intrínseco de la igualdad con el valor intrínseco de la justicia proporcional, siendo el mérito un tipo de principio de justicia proporcional.

Antes de entrar en la discusión de manera más detallada es necesario hacer una breve precisión metodológica. Como veremos, la discusión se desarrolla en torno a casos o ejemplos elaborados con el fin de respaldar intuiciones. Es por esta razón que la discusión de esas intuiciones implica el análisis de los casos usados para respaldarlas. Esto explica que, en las páginas que siguen, la discusión tenga ese carácter. Es posible que la naturaleza misma del problema -i.e., el valor intrínseco de la igualdad- requiera que las respectivas posiciones se defiendan de esa manera. Si bien este punto es importante y requiere de mayor discusión, parece razonable proceder aceptando los términos en los que de hecho la discusión se ha venido realizando.

\section{El caso de las cumbres gemelas}

Shelley Kagan (1999, 306-8) ha elaborado dos casos con el propósito de cuestionar el valor intrínseco de la igualdad. Antes de describir estos casos es necesario introducir ciertas distinciones que nos ayudarán a evaluar mejor el peso intuitivo que eventualmente puedan tener. Los casos en cuestión son Las cumbres gemelas y Las cumbres gemelas revisado. En ambos títulos el término «cumbre» refiere al punto en el que una persona tiene exactamente lo que se merece. Esto es lo que Kagan llama mérito absoluto. Este tipo de mérito se distingue del mérito comparativo (Kagan 1999, 301). Según el mérito comparativo, si A es igualmente merecedor que $\mathrm{B}$ en términos absolutos, entonces A debe estar tan bien como B. Esto significa que importa, desde el punto de vista del mérito comparativo, cómo A está en relación con $\mathrm{B}$, considerando que tan absolutamente merecedores son ambos. Tanto el mérito absoluto como el comparativo deben distinguirse, a su vez, del mérito especifico (Ibid., p. 302). Si, por ejemplo, C merece menos que $\mathrm{D}$ en términos absolutos - es decir, su cumbre es de menor altura que la de D - pero está más lejos de su cumbre que D de la suya, entonces C es específicamente más merecedor que $\mathrm{D}$. La razón que justifica este juicio es que, mientras más lejos está una persona de su cumbre, mayor es el impacto de cada 
unidad adicional del bien relevante. Esto es lo que Kagan llama mérito curvo [«curved desert»] $(1999,301)$. No es relevante para nuestros propósitos evaluar la plausibilidad de estas distinciones porque el tipo de igualdad que nos interesa aquí supone aceptar, al menos, que el mérito tiene tanto una dimensión comparativa como una dimensión no-comparativa, de lo contrario no tendría sentido que se hablara de desigualdades inmerecidas. Si caracterizamos una desigualdad como inmerecida esto significa que creemos que el mérito tiene una dimensión comparativa, esto es, que nos preocupa el que algunos individuos tengan menos de lo que merecen en comparación con otros. ${ }^{6}$ Esto significa que, en términos generales, las distinciones propuestas por Kagan no deberían ser problemáticas para quienes conciben la igualdad en términos restringidos. Para nuestros propósitos, las nociones especialmente relevantes son las de mérito comparativo, mérito absoluto $\mathrm{y}$, en menor medida, mérito específico.

Describamos ahora el primero de los casos con los que Kagan pretende poner en cuestión el valor intrínseco de la igualdad.

Las cumbres gemelas: ${ }^{7}$

1. Imaginemos un mundo de dos personas, A y B.

2. A es mucho menos merecedor que B. (Imaginemos, por ejemplo, que A es un pecador y $\mathrm{B}$ es un santo).

3. A está mejor de lo que debería - es decir, está más allá de su cumbre.

4. B está peor de lo que debería - es decir, está bajo su cumbre.

5. A está peor que B.

Si podemos ayudar sólo a $\mathrm{A}$ o a $\mathrm{B}$, ¿a quién deberíamos ayudar? Ya que A está peor que B, la igualdad demanda ayudar a A. Pero, ya que B está bajo su cumbre y A sobre la suya, el mérito demanda ayudar a B. Parece ser intuitivamente claro, según Kagan, que deberíamos ayudar a B. Si ayudáramos a A esto implicaría dejar a A mucho mejor de lo que merece y eso parece, al menos, más injusto que dejar a A como está. Es importante notar que en este caso, cuando se habla de las demandas de la igualdad, se está interpretando la igualdad en términos estrictos. Recordemos que hablamos de igualdad estricta cuando nos preocupa la igualdad independientemente del mérito, es decir, cuando creemos que la igualdad es importante en sí misma. Este caso busca mostrar precisamente que no parece ser intuitivamente plausible que la igualdad estricta importe más que el mérito.

Con el fin de evaluar la posibilidad de que la igualdad importe en algún otro sentido Kagan elabora una nueva versión de este caso. La igualdad podría importar cuando la consideramos no-estrictamente, es decir, quizás la igualdad importa pero sólo entre personas igualmente merecedoras. Este es - con ciertas restricciones - el modo en el cual el igualitarismo de la suerte ha interpretado la noción de igualdad. El propósito ahora es elaborar un caso en el que el mérito sea neutral respecto de ayudar tanto a quien está mejor como a quien está peor. $\mathrm{Si}$, a pesar de dicha neutralidad, creemos que siguen existiendo razones que demandan ayudar al que está peor, esto apoyaría la idea de que la igualdad noestricta importa en sí misma. Es necesario notar que, cuando se habla aquí de «igualmente merecedoras», no se está diciendo que, para examinar si la igualdad importa, las personas tienen que merecer lo mismo en términos absolutos, sino que deben ser igualmente merecedoras en términos específicos. Eso es lo que permite, de hecho, que se pueda elaborar un caso en el que el mérito sea 
neutral respecto de ayudar tanto a quien está mejor como a quien está peor. Revisemos, entonces, esta nueva versión.

Las cumbres gemelas revisado:

1. Imaginemos un mundo de dos personas, A y B.

2. A es ligeramente menos merecedor que B.

3. A está peor que B.

4. A tiene menos de lo que merece y B tiene menos de lo que merece.

5. Tanto A como B tienen menos de lo que merecen por la misma cantidad, esto es, están a la misma distancia de su cumbre de mérito.

El principio del mérito es indiferente entre ayudar a A o ayudar a B pues ambos tienen menos de lo que merecen por la misma cantidad, es decir, ambos merecen lo mismo específicamente. El igualitarismo no-estricto podría favorecer a $\mathrm{A}$, que está peor que B pero, según Kagan, no hay razón para ayudar a A porque las demandas, tanto de A como de B, son igualmente fuertes: ${ }^{8}$ ambos están a la misma distancia de sus cumbres. Entonces, el caso de Las cumbres gemelas revisado mostraría que la igualdad noestricta - es decir, la igualdad entre personas igualmente merecedoras - no tiene valor intrínseco pues, si lo tuviera, habrían razones para corregir la desigualdad entre A y $\mathrm{B}$, razones normativamente más poderosas que las razones que ofrece el mérito para dejar las cosas como están.

El caso de Las cumbres gemelas pretende mostrar que la igualdad estricta pesa menos - normativamente hablando- que el mérito y el caso de Las cumbres gemelas revisado pretende mostrar que la igualdad no-estricta pesa menos -normativamente hablando- que el mérito. La conclusión es ciertamente importante pues pone en cuestión el carác- ter igualitario de las teorías que introducen el mérito como un criterio legitimador de ciertas desigualdades. En la medida en que se asuma que existe una conexión entre mérito y responsabilidad, de modo que se entienda que las desigualdades que no son responsabilidad de las personas son desigualdades inmerecidas, la conclusión recién formulada incluye también al igualitarismo de la suerte.

\section{Desigualdades inmerecidas e igualdad}

Si la igualdad restringida no tiene valor intrínseco, entonces la preocupación por las desigualdades que no son responsabilidad de las personas debe entenderse, en realidad, no como una preocupación por la igualdad, sino por el mérito. Desde luego, este es un punto importante pues muestra que lo que nos parece injusto no es la desigualdad sino que las personas no tengan lo que merecen comparativamente.

Serena Olsaretti $(2002,395)$ recientemente ha elaborado una réplica a esta conclusión. Esta réplica tiene la forma de un caso hipotético con el que se busca mostrar que la igualdad restringida sí tiene valor intrínseco. Describamos primero el caso hipotético de Olsaretti y luego pasemos a revisar su plausibilidad.

Imaginemos dos mundos. En el primero los individuos son igualmente merecedores y están igualmente bien. En el segundo los individuos no son igualmente merecedores y no están igualmente bien. Ninguna consideración sobre el mérito podría llevarnos a preferir un mundo por sobre otro porque en ambos los individuos tienen lo que se merecen. En el primer mundo hay igualdad merecida y en el segundo hay desigualdad merecida. Pero, dado que en el primer mundo (M1) no hay desigualdad, Olsaretti piensa que esta característica hace a 
este mundo - al menos en un sentidomejor que el segundo (M2). Esto significa que la igualdad sí tiene valor intrínseco - o, más exactamente, Olsaretti cree que los individuos que piensan que la igualdad tiene valor intrínseco afirmarían que M1 es, al menos en un sentido, mejor que M2 porque en M1 no hay desigualdad - El caso es interesante - esto significa que hay, al menos, una buena razón para discutirlo aquí- porque, desde el punto de vista del mérito, ambos mundos son igualmente justos — pues tanto en M1 como en M2 el estado de cosas es merecido- pero se pretende mostrar, sin embargo, que, a pesar de eso, la igualdad agregaría algo más.

No me parece que esta manera de defender el valor intrínseco de la igualdad sea plausible. Según Olsaretti, un mundo puede ser mejor que otro en términos de justicia incluso si en ambos mundos los individuos tienen lo que se merecen. ¿Por qué la desigualdad en M2 es mala, o por qué la igualdad en M1 es buena si en ambos casos el estado de cosas es merecido? ¿En qué sentido la desigualdad merecida contribuye a hacer M2 - al menos bajo un aspecto- peor que M1? Si es que lo que importa es la existencia de igualdad o desigualdad merecida, no parece haber razón para afirmar que M1 es, en un sentido, mejor que M2. En M1 existe igualdad merecida y en M2 existe desigualdad merecida, lo que significa que, desde el punto de vista de la justicia, ambos mundos son igualmente justos porque en ambos mundos los individuos tienen lo que se merecen. ${ }^{9}$ La conclusión que quisiera defender en relación a este punto es la siguiente: cuando nos importan las desigualdades inmerecidas, si el principio del mérito es satisfecho en el estado de cosas $A$ y en el estado de cosas $B$, el estado de cosas $A$ no puede ser más justo que el estado de cosas $B$ y viceversa.
Es decir, cuando dos estados de cosas no tienen desigualdades inmerecidas ambos son igualmente justos (independientemente de cuánta desigualdad merecida exista).

$\mathrm{Si}$, contra lo afirmado en la conclusión, alguien cree que M1 es más justo que M2, la justificación de ese juicio debería incluir más factores que la mera constatación de la existencia de desigualdades inmerecidas, pues la eventual eliminación de las desigualdades inmerecidas no bastaría para hacer de un estado de cosas un estado justo - si bastase no habría razón para creer que M1 es más justo que M2. ${ }^{10}$ No quisiera aquí aventurarme a pensar cuáles podría ser esos otros factores. Para nuestros propósitos es suficiente establecer que, quien cree que M1 es más justo que M2, no debe apelar sólo a la existencia de desigualdades inmerecidas como el foco de sus preocupaciones distributivas sino que a algo más. El tipo de igualitarismo noestricto que estamos discutiendo aquí justamente no hace eso.

\section{El eslogan y el valor intrínseco de la igualdad}

La conclusión establecida en la sección anterior respalda la siguiente idea: cuando nos importa la igualdad no-estricta lo que nos importa no es la igualdad sino que las personas tengan lo que merecen. La dimensión comparativa del mérito puede hacernos creer que lo relevante es la igualdad cuando, en realidad, ésta no es más que uno de los posibles resultados de la aplicación del principio del mérito - es decir, personas igualmente merecedoras en términos absolutos deberían estar igualmente bien (en términos del bien relevante) y, sólo en esos casos, la igualdad debería parecernos moralmente deseable. Esta es una cuestión especialmente importante que retomaré hacia el 
final de este trabajo. La menciono aquí porque ayuda a entender en qué sentido la discusión del caso hipotético de Olsaretti resulta instructiva.

Analicemos ahora otro modo de defender el valor intrínseco de la igualdad que tampoco parece tomar en cuenta la distinción entre las demandas propias de la igualdad y las demandas propias del mérito comparativo. Esta defensa sostiene que, ya que existen casos en los que la eliminación de la desigualdad es buena incluso cuando no es buena para nadie, la igualdad tiene valor intrínseco. Por ejemplo, una desigualdad puede ser eliminada nivelando hacia abajo, esto es, empeorando la condición de una persona sin mejorar la condición de nadie. Algunos anti-igualitarios piensan que nivelar hacia abajo no puede hacer un estado de cosas mejor. Ellos creen que, ya que nivelar hacia abajo no es bueno para nadie, no hay nada bueno en eso. Esto se conoce como la objeción de la nivelación hacia abajo y es comúnmente usada como una objeción contra quienes defienden el valor intrínseco de la igualdad. ${ }^{11}$ Esta objeción está dirigida contra el valor intrínseco de la igualdad porque, si es exitosa, mostraría que consideraciones de bienestar son, en este caso, más relevantes que la igualdad y que, por tanto, no hay nada bueno en nivelar hacia abajo.

Larry Temkin piensa que la fuerza de esta objeción descansa en la fuerza de lo que él llama El eslogan (E). Según E «una situación no puede ser peor (o mejor) que otra si es que no hay nadie para quien es peor (o mejor)» (2000, p. 132). Dado que nivelar hacia abajo no es bueno para nadie, no deberíamos decir que la igualdad así generada contribuye a la bondad de un estado de cosas. De hecho, deberíamos decir que una igualdad conseguida mediante una nivelación hacia abajo hace que el estado de cosas sea peor, ya que la nivelación es peor para alguien y mejor para nadie.

Temkin cree que deberíamos rechazar tanto E como la objeción de la nivelación hacia abajo. Existen algunos ejemplos, según Temkin, que muestran que $\mathrm{E}$ es falso y que, en consecuencia, la igualdad tiene valor intrínseco. Estos ejemplos son el problema de la no-identidad, la paradoja de la mera adición, y el ejemplo de los santos y los pecadores. Los dos primeros ejemplos fueron elaborados por Derek Parfit (1984, caps. 16 y 19) y el tercero fue elaborado por Shelly Kagan $(1999,304)$. No discutiré todos estos ejemplos aquí porque, incluso si ellos mostraran que E es falso, eso no implica que ellos también muestren que la igualdad tiene valor intrínseco y que, como consecuencia, el tipo de nivelación hacia abajo asociado a ella, es, al menos bajo un aspecto, bueno. Por ejemplo, decir que, en algunos casos, nivelar hacia abajo hace un estado de cosas mejor porque eso es lo que la justicia demanda, no es una manera de mostrar que la igualdad tiene valor intrínseco. Podrían existir razones para nivelar hacia abajo que están relacionadas, por ejemplo, con el mérito. De hecho, uno de los ejemplos que Temkin más discute es el de los santos y pecadores elaborado por el propio Kagan. Si un pecador está mejor que un santo y tú puedes dejar las cosas como están o nivelar hacia abajo al pecador para que esté en la condición del santo, hay al menos una razón para hacer esto último. Pero, si la igualdad no es lo mismo que el mérito - como Temkin lo admite ${ }^{12}$ - este ejemplo no muestra que la igualdad tenga un valor intrínseco. Sólo muestra que, en relación al mérito, E es falso - esto es, muestra que la contribución del mérito a la bondad de un estado de cosas no está relacionada con el hecho de que el mérito sea bueno para alguien. (Una prueba de esto está en que, cuando 
una persona tiene más de lo que merece, este hecho hace que el estado de cosas sea peor). Es importante remarcar este punto porque los ejemplos de Temkin relativos a la falsedad de $\mathrm{E}$ son dados en el contexto de la discusión del valor intrínseco de la igualdad restringida $\mathrm{y}$, como ya lo hemos dicho, el interés central de este tipo de igualitarismo son la desigualdades inmerecidas. Si no se distingue claramente entre el mérito y la igualdad cuando se discute acerca de la falsedad de E, no seremos capaces de separar los argumentos que defienden el valor intrínseco de la igualdad de aquellos que defienden el valor intrínseco del mérito.

Del ejemplo de los santos y los pecadores podemos extraer la siguiente conclusión: la existencia de razones para nivelar hacia abajo no nos debe llevar a concluir que la igualdad tiene valor intrínseco. Antes de concluir algo como eso debemos examinar el tipo de razones que podrían justificar nivelar hacia abajo.

En el ejemplo de los santos y los pecadores las razones que justifican nivelar hacia abajo no dicen relación con la igualdad sino que con el mérito - específicamente, con el mérito comparativo-. Aunque parezca sorprendente, esta confusión no ha sido notada en la discusión reciente sobre el valor de la igualdad. ${ }^{13}$ Este es un punto relevante si atendemos, además, a la especial relevancia que los artículos de Temkin sobre la igualdad han tenido en la discusión contemporánea.

Esta misma confusión puede apreciarse en otros dos ejemplos que Temkin ofrece para defender el valor intrínseco de la igualdad. Dada la importancia de la cuestión puede resultar ilustrativo detenerse un momento en estos ejemplos. El primero es el caso de un mundo A donde la mitad son ciegos y un mundo B donde todos lo son $(2000,131,136)$. Temkin piensa que, si creemos que la igualdad tiene valor intrínseco, también deberíamos pensar que hay al menos una razón para transformar A en B. El otro ejemplo es el caso de ciertas personas que accidentalmente dan con un fruto raro que los hace inmortales. Entonces, «mientras algunas personas mueren, otras viven para siempre» $(2003,781)$. En el primer ejemplo hay desigualdad en lo que refiere a la vista y en el segundo hay desigualdad en lo que refiere a la vida y la muerte. El punto relevante debería ser que, en ambos casos, la desigualdad importa porque es inmerecida. Si no fuera esto lo que importa, entonces no estaríamos adhiriendo a la igualdad no-estricta, sino que a la igualdad estricta. Ya que Temkin adhiere a la primera y no a la segunda, debemos interpretar estos ejemplos en esos términos. Creo que, cuando es esto lo que importa, estos no son buenos ejemplos acerca del valor intrínseco de la igualdad porque, si el principio del mérito fuera satisfecho, no existirían desigualdades inmerecidas $\mathrm{y}$, por tanto, las desigualdades en cuestión no serían injustas. Es decir, si, por diversas razones, la mitad ciega del mundo A fuera ciega por razones debidas a su responsabilidad, la desigualdad en cuestión no nos parecería relevante desde el punto de vista de la justicia. $\mathrm{Si}$ —en el segundo ejemplolas personas que no se hacen inmortales tienen responsabilidad en no haber comido del fruto que, suponemos, sabían que los haría inmortales, entonces la desigualdad en cuestión no es relevante desde el punto de vista de la justicia. Si esto es verdadero, entonces, que nos importe la existencia de desigualdades inmerecidas se explica única y exclusivamente por que nos importa la no satisfacción del principio del mérito.

De la discusión de los ejemplos de Temkin es posible obtener la siguiente 
conclusión: la no satisfacción del principio del mérito podría implicar la existencia de desigualdad inmerecida o igualdad inmerecida. Lo relevante, entonces, no es que un estado de cosas sea igual o desigual, sino que sea inmerecido - es decir, que por lo menos uno de los miembros de ese estado de cosas no tenga lo que se merece-. Por tanto, creer que las desigualdades (o igualdades) inmerecidas son injustas es creer que lo que la justicia requiere es la satisfacción del principio del mérito.

Pero si el mérito y la igualdad noestricta son diferentes - como lo supone Temkin-, esta diferencia debería tener un impacto normativo. En los ejemplos vistos arriba el impacto normativo de la igualdad es cero porque todo lo que necesitamos para lidiar con las desigualdades inmerecidas de estos ejemplos es satisfacer el principio del mérito: cuando las personas tienen lo que se merecen no hay desigualdades inmerecidas. Esto muestra que Temkin está aquí usando dos etiquetas distintas para un mismo problema, haciéndonos suponer que existe, por un lado, la cuestión de las desigualdades inmerecidas y, por el otro, la cuestión de las personas que no tienen lo que se merecen. Pienso que estas no son dos cuestiones diferentes sino, en el mejor de los casos, dos maneras de mirar un mismo problema: las consecuencias comparativas de la no satisfacción del principio del mérito, es decir, que existan personas que están peor que otras porque no tienen lo que se merecen.

Creo que lo que explica esta confusión es no distinguir adecuadamente entre la dimensión comparativa del mérito y la igualdad. Cuando nos interesa la desigualdad en la medida en que es inmerecida, la igualdad es simplemente uno de los estados de cosas posibles derivados de la aplicación del principio del mérito. La eliminación de una desigual- dad inmerecida implicará la existencia de igualdad únicamente entre sujetos que merecen lo mismo, pero también puede implicar la existencia de desigualdad entre sujetos que no merecen lo mismo.

\section{Igualdad de base}

Los argumentos ofrecidos contra Temkin y Olsaretti pretenden mostrar que, en relación con la igualdad no-estricta, el mérito no puede oponerse nunca a la igualdad, de manera que no es correcto hablar en términos de la (posible) oposición entre mérito e igualdad. ${ }^{14}$ La razón que explica esto es simple. Si la igualdad se opusiera al mérito - es decir, si pesara normativamente más que (o tanto como) el mérito- no sería verdadero que la igualdad importa sólo entre personas igualmente merecedoras y, como sabemos, en esto consiste precisamente la igualdad no-estricta. En términos del igualitarismo de la suerte el mismo punto podría ponerse de la siguiente manera. Si la igualdad se opusiera al mérito no sería verdadero que la igualdad nos importa porque nos importan las desigualdades que no son responsabilidad de las personas sino de la suerte (es decir, desigualdades inmerecidas).

Pero quizás la igualdad tiene valor intrínseco porque, antes que los individuos se vuelvan desigualmente merecedores debido a sus ejercicios comparativos de responsabilidad —los recién nacidos, por ejemplo - todos tienen una legítima demanda a una igual cantidad del bien relevante que no puede estar basada en el mérito sino en la igualdad. Ese sería el tipo de igualdad que tendría valor intrínseco. Una igualdad que podríamos llamar igualdad de base. Ya que los recién nacidos no están en condiciones de des plegar acciones que los hagan merecedores de algo, el mérito es inaplicable en este estadio. Entonces, si es verdad 
que existe igualdad de base entre los recién nacidos, esta igualdad no puede estar basada en el mérito. Este es un punto ciertamente importante pero no es necesario que lo abordemos aquí de manera acabada. Lo que quisiera hacer a continuación es bosquejar una objeción posible a esta manera de defender el valor intrínseco de la igualdad.

Partamos por notar que, si la igualdad tiene valor intrínseco en este sentido, entonces no es éste el mismo sentido en el cual la expresión fue usada cuando discutíamos acerca del peso normativo de la igualdad versus el peso normativo del mérito. En ese caso nuestra discusión se centraba en examinar el valor de la igualdad entre personas que eventualmente podían ser desigualmente merecedoras en virtud de sus decisiones responsables. Esto significa que existen al menos dos argumentos distintos a favor del valor intrínseco de la igualdad y que el argumento relativo a la base igualitaria no puede ser usado para responder a mi crítica relativa al valor intrínseco de la igualdad elaborada en las secciones IV y V. Además, esto sugiere que la igualdad podría no tener valor intrínseco según el primer sentido pero sí podría tener valor intrínseco según el segundo sentido $\mathrm{y}$ viceversa.

Examinemos brevemente por qué el mérito no podría justificar la base igualitaria y por qué creo que eso no implica que la igualdad tenga valor intrínseco. $\mathrm{Si}$ quisiéramos argumentar a favor del mérito como posible justificación de las base igualitaria, deberíamos mostrar que no es necesario para una base del mérito que ésta sea algo que una persona despliega. Esto significa que una persona puede merecer algo incluso si no despliega ninguna base de mérito. El obvio problema con esta idea es que, si no es necesario que una persona despliegue una base del mérito para merecer algo, enton- ces una persona puede merecer algo sin ser responsable de aquello que la hace merecedora de ese algo. El tipo de igualitarismo no-estricto que estamos discutiendo en este artículo trabaja con la convicción de que el mérito «rastrea» la responsabilidad, es decir, que para ser merecedora de algo una persona tiene que ser responsable de lo que la hace merecer ese algo. Es justamente por esa razón que los defensores de la igualdad no-estricta son sensibles a las desigualdades generadas por el impacto de la suerte en la vida de las personas e insensibles a las desigualdades derivadas de la responsabilidad de las personas. Las desigualdades inmerecidas son el resultado del impacto diferencial de la suerte y las desigualdades merecidas son el resultado del ejercicio comparativo de la responsabilidad de las personas. ${ }^{15}$ Entonces, si el mérito rastrea la responsabilidad y lo que justifica la base igualitaria no es algo que la persona despliega, el mérito no puede justificar la base igualitaria. Podría pensarse que esto es una especie de imposibilidad conceptual: cuando tratamos de justificar una base igualitaria, la responsabilidad no puede ser parte de esa justificación porque, ya que lo que justifica la base no es algo que puede ser desplegado - como lo muestra el caso de los recién nacidos-, no podemos decir que todos merecen una igual cantidad de bienestar porque todos son igualmente responsables. Si lo que justifica la base fuese algo que pudiese ser desplegado, entonces no estaríamos hablando de una base, es decir, de algo que es anterior al hecho de que las personas se vuelvan (de manera legítima) desigualmente merecedoras.

Pero, si esta imposibilidad conceptual es real, entonces no puede decirse que la base igualitaria se fundamenta en la igualdad en lugar del mérito. No es apropiado decir — como una manera de 
mostrar que la igualdad justifica las base igualitaria - que «antes de ser desigualmente merecedores, los individuos tienen derecho a [are entitled to] estar igualmente bien porque cualquier desigualdad entre ellos es inmerecida, y la igualdad requiere la rectificación de las desigualdades inmerecidas» (Olsaretti 2002, 397). Si el mérito no puede ser aplicado en un estadio de línea de base porque los individuos son incapaces de desplegar base de mérito alguna, entonces el concepto de desigualdades inmerecidas tampoco puede ser aplicado en ese estadio. Que el mérito sea inaplicable en un cierto dominio debería significar que las desigualdades merecidas $o$ inmerecidas son algo que no pueden existir en ese dominio. No hay razón para creer que el mérito es inaplicable al nivel de la línea de base y, a pesar de eso, describir las desigualdades en cuestión como inmerecidas. Entonces, si no es correcto hablar de desigualdades inmerecidas a nivel de línea de base, el tipo de igualdad que está en juego en ese estadio no es el tipo de igualdad que nos interesa aquí. Además, estoy de acuerdo que, si el mérito rastrea la responsabilidad, el mérito es inaplicable al nivel de línea de base. Pero admitir eso no es un problema para lo que pretendo afirmar en este trabajo -i.e., que la igualdad (no-estricta) no tiene valor intrínseco- porque, si el mérito es inaplicable, la igualdad no-estricta también lo es. ${ }^{16}$

\section{Conclusión}

Podemos concluir entonces que los argumentos relativos, por un lado, a la contribución de la igualdad a la bondad de un estado de cosas y, por el otro, al rol justificador de la igualdad respecto de la base igualitaria son ambos deficientes en tanto que argumentos a favor del valor intrínseco de la igualdad. De la discusión del primer argumento se puede extraer una lección importante: cuando la igualdad es entendida como igualdad no-estricta la preocupación central es por las consecuencias comparativas de la no satisfacción del principio del mérito. Esto muestra que, cuando la igualdad es entendida de manera no-estricta, no hay un principio de igualdad, por un lado, y un principio del mérito, por el otro. Cuando separamos igualdad y mérito debemos interpretar la igualdad como igualdad estricta - sólo en este caso hay igualdad $y$ mérito- Cuando interpretamos la igualdad como igualdad no-estricta nuestra preocupación central no es de corte igualitario sino una preocupación relativa a que las personas tengan lo que se merecen. La existencia de desigualdades inmerecidas es sólo un tipo de consecuencia posible que se deriva del que las personas no tengan lo que se merecen. La otra consecuencia posible es la existencia de una igualdad inmerecida. Si la igualdad «es una porción de nuestra preocupación sobre la equidad (fairness) que se focaliza en cómo las personas están en relación con otras» (Temkin 2003, 767), no parece haber razón para no prestar atención a la existencia de la igualdad inmerecida. Una igualdad inmerecida es injusta, así como es injusta una desigualdad inmerecida.

$\mathrm{Si}$ es que estoy en lo correcto en este punto, esto significa que no es acertado decir, por un lado, que la igualdad «requiere la rectificación de las desigualdades por las que los individuos no son responsables» (Olsaretti 2002, 399) y, por el otro, que el mérito «requiere que las desigualdades por las cuales los individuos son responsables sean preservadas» (ibid.). La existencia de desigualdades por las cuales los individuos no son responsables necesariamente implica la existencia de individuos que no tienen lo que se merecen y, por esta razón, es per- 
fectamente plausible afirmar que el principio del mérito requiere la rectificación de esta situación, y no sólo la preservación de las desigualdades merecidas. Un estado de cosas en el cual hay desigualdad inmerecida puede ser descrito en términos de una desigualdad por la cual los individuos no son responsables y el mismo estado de cosas - puede ser descrito en términos de personas que no tienen lo que se merecen. Que existan dos descripciones posibles no implica la existencia de dos cuestiones normativas diferentes: la cuestión de la igualdad y la cuestión del mérito. La cuestión de la igualdad es, en parte - i.e., no sólo- la cuestión del mérito, porque la existencia de desigualdades inmerecidas es una posible consecuencia de la no satisfacción del principio del mérito.

Quisiera concluir retomando lo que señalé al comienzo de la sección $\mathrm{V}$. La dimensión comparativa del mérito - es decir, la existencia de desigualdades inmerecidas - puede hacernos creer que la igualdad tiene valor intrínseco. Pero la igualdad debería ser entendida como uno de los posibles resultados de la aplicación del principio del mérito - es decir, personas igualmente merecedoras en términos absolutos deberían estar igualmente bien (en términos del bien relevante) y, sólo en esos casos, la igualdad debería parecernos moralmente deseable- - Si los argumentos ofrecidos contra el valor intrínseco de la igualdad son correctos, la igualdad es valiosa de manera de hacer que la nivelación hacia abajo sea, bajo al menos un aspecto, buena. Pero la razón de esto no radica en que la igualdad tenga valor intrínseco. Más bien, la razón está en que la igualdad es un sub-producto de lo que es intrínsecamente valioso: la justicia proporcional. El principio del mérito es una manera de entender la justicia proporcional. Cuando cada uno tiene lo que se merece, cada uno tiene lo que tiene en proporción al mérito desplegado. Los argumentos desarrollados aquí contra el valor intrínseco de la igualdad permiten establecer esta importante conclusión. Debo conformarme aquí simplemente con señalarla. Un desarrollo adecuado de este punto debería ser materia de otro trabajo. ${ }^{17}$

\section{BIBLIOGRAFÍA}

Anderson, E. S. (1999): "What is the Point of Equality». En Ethics, 99, 2, 287-337.

Arneson, R., (1989): «Equality and Equal Opportunity for Welfare», Philosophical Studies, 56, 77-93.

Carter. I y Page, O., (2011): «Where is the Value in Equality?» (manuscrito).

Cohen, G. A., (1989): «On the Currency of Egalitarian Justice», Ethics, vol. 99, 4, 906-44.

Crisp, R. (2003): «Equality, Priority, and Compassion», Ethics, 113, 4, 745-63.

Dworkin, R. (2000): Sovereign Virtue. The Theory and Practice of Equality, Harvard University Press, Cambridge, Mass.

Kagan, Shelly (1999): «Equality and Desert», in What Do We Deserve? A Reader on Justice and Desert. Pojman, L. P. and McLeod, O (eds.), New York and Oxford: Oxford University Press.

Feinberg, J., (1970): «Justice and Personal Desert», Doing and Deserving, Princeton University Press, Princeton, 55-94.

Nagel, T., (1991): Equality and Partiality, Oxford University Press, New York.

Olsaretti, Serena (2002): «Unmasking Equality? Kagan on Equality and Desert», Utilitas, vol. 14, n. ${ }^{\circ}$ 3, 387-400. 
Page, O., (2007): «Igualdad, suerte y responsabilidad«, Estudios Públicos, 106, 153-174.

Parfit, Derek, (1991): «Equality or Priority?», Tanner Lectures, University of Kansas.

Scheffler, S., (2003):«What is Egalitarianism?», Philosophy and Public Affairs, 31, 1, 5-39.

Sher, G., (1987): Desert, Princeton University Press, Princeton.
Spector, Ezequiel (2011): «Do you Deserve to be Talented?», Utilitas, vol. $23,{ }^{\circ}{ }^{\circ} 1,115-125$.

Temkin, Larry (2000): «Equality, Priority, and the Leveling Down Objection», in The Idea of Equality, Clayton, M. and Williams, A. (eds.), New York: Palgrave MacMillan.

Temkin, Larry (2003): «Egalitarianism Defended», Ethics, vol. 113, n. ${ }^{\circ}$ 4, 764-782.
1 Este trabajo forma parte del proyecto Fondecyt N. ${ }^{\circ} 1100978$.

2 La expresión «igualitarismo de la suerte» la tomo de Anderson (1999).

${ }^{3} \mathrm{He}$ analizado alguno de esos problemas en Page (2007).

4 Respuestas a esta pregunta se pueden encontrar, por ejemplo, en Arneson (1989), Cohen (1989), Dworkin (2000).

5 Algunos de estos problemas se encuentran bien expuestos en el ya clásico ensayo de Feinberg (1970). Véase también el tratamiento pionero de Sher (1987).

${ }^{6}$ Algunas de las complejidades de la dimensión comparativa del mérito se pueden encontrar en Kagan (1999, 301-3).

7 Kagan presenta estos casos usando gráficos (esos gráficos justifican el que se hable de «cumbres»). He modificado esa presentación con el fin de hacer las cosas más simples.

8 Además, si A es un pecador y $\mathrm{B}$ un santo, Kagan piensa que la demanda de B es más fuerte que la de A $(1999,308)$.

9 No estoy con esto señalando que la justicia debería ser entendida así. Estoy diciendo que, si la entendemos así, entonces no parece haber razón que justifique creer que M1 es mejor que M2 en términos de justicia.
10 Evidentemente muchos otros factores no relacionados con la igualdad distributiva son relevantes para evaluar qué tan justo es un estado de cosas todo considerado. Ya que nuestro objetivo es el de examinar la coherencia del igualitarismo no-estricto como interpretación posible de la igualdad distributiva, esos otros factores no deben ser usados para examinar en qué otro sentido M1 podría ser más justo que M2.

11 Derek Parfit (1991, 81-125).

12 Temkin (2003, 779): «Why shouldn't we also care about whether moral agents get what they deserve (justice), or about how individuals fare relative to others (equality), or about whether rational agents have acted freely, autonomously, or morally?» (énfasis mío). Véase también $(2003,780)$ donde Temkin distingue entre mérito y equidad comparativa.

13 Algunos de los argumentos de Temkin contra $\mathrm{E}$ han sido discutidos en detalle, por ejemplo, por Crisp (2003). En dicha discusión no hay argumento alguno que apele a la confusión a la que estoy haciendo mención.

14 Sí es correcto hablar en términos de oposición cuando interpretamos la igualdad como igualdad estricta. 
15 Cuando hablo de «impacto diferencial de la suerte» me refiero a desigualdades debidas a la suerte.

${ }^{16}$ Podría replicarse que este es un problema meramente semántico y que, si bien no es correcto hablar de desigualdades inmerecidas a nivel de línea de base, podría hablarse de desigualdades no-merecidas. Se dice que una desigualdad es no-merecida cuando no es posible hacer algo para merecerla o no merecerla. Un ejemplo es una capacidad natural como la visión. La desigualdad entre quienes nacen sin poder ver y quienes ven es nomerecida porque no era posible para los afectados hacer algo para evitar su condición. Por esta razón no tiene sentido afirmar que la ceguera es inmerecida. Para que algo sea inmerecido para una persona debe ser verdadero que ésta hizo algo —o dejó de hacer algo que podría haber hecho- para no merecerlo. Si la ceguera de nacimiento no está relaciona- da con la responsabilidad, no es adecuado referirse a ella como algo inmerecido. En relación con la diferencia entre no-merecido e inmerecido véase Spector (2011). Parte de la justificación de esta distinción está relacionada con el siguiente razonamiento: «if it does not make sense to ask what people did before their birth, it does not make sense to ask whether people deserve their inborn characteristics either» (Spector 2011, 120). Creo que esta distinción es correcta pero no creo que pueda ser usada como una réplica a mi crítica sobre el valor intrínseco de la igualdad de base. Si lo que la igualdad persigue a nivel de línea de base es eliminar desigualdades no-merecidas, entonces no es el tipo de igualdad cuyo valor intrínseco hemos estado discutiendo a lo largo de este trabajo.

17 Un desarrollo más amplio sobre este punto se encuentra en Carter y Page (2011). 